\title{
HOMOLOGY COMODULES
}

\author{
BY \\ ARUNAS LIULEVICIUS( ${ }^{1}$ )
}

Let $X$ be a topological space, $H^{*}(X)$ the cohomology of $X$ with $Z_{2}$ coefficients, $A$ the Steenrod algebra [4] over $Z_{2}$, then $A$ acts on $H^{*}(X)$ :

$$
\mu: A \otimes H^{*}(X) \rightarrow H^{*}(X)
$$

If we denote by $A_{*}$ the graded dual of $A, H_{*}(X)$ the homology of $X$ over $Z_{2}$, then

$$
\mu_{*}: H_{*}(X) \rightarrow A_{*} \otimes H_{*}(X)
$$

makes $H_{*}(X)$ into a comodule over $A_{*}$. Since $A_{*}$ is much easier to handle than $A$, it is important to know $\mu_{*}$. In this note we describe $\mu_{*}$ for $X=B G$ and $X=M G$, where $G=O(n), U(n), S p(n), O, U, S p$.

We remark that Van de Velde [5] determines the coaction for all primes $p$ via an elegant recursive formula involving divided powers. This note describes another algorithm for obtaining the components of the coaction by means of an electronic computer. The algorithm works only for $p=2$.

The paper is organized as follows: the first section describes the initial argument by Van de Velde [5], the second section gives a few immediate results for the coaction in $H_{*}\left(R P^{\infty}\right)$, the third gives the computer results on $\mu_{*}\left(x_{32}\right)$, the fourth points out the implications for the homology of classifying spaces and Thom spaces.

1. Main formula. Let $A$ be a graded connected biassociative Hopf algebra (in the application it will be the mod 2 Steenrod algebra) over a field $F$. Let $M$ be a graded left Hopf algebra over $A$ [2]: that is, $M$ is a left $A$-module with action

$$
\mu: A \otimes M \rightarrow M,
$$

and multiplication

$$
m: M \otimes M \rightarrow M,
$$

such that the "Cartan formula" is valid-the following diagram is commutative

Received by the editors August 9, 1966 and, in revised form, June 29, 1967.

(1) The author was partially supported by NSF grant GP-3936 during the preparation of this paper. 


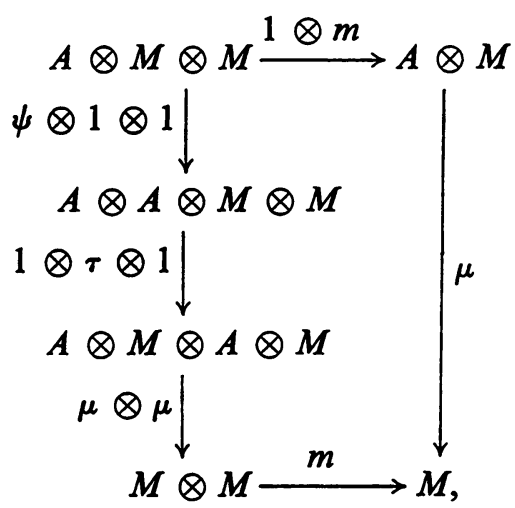

where $\psi$ is the coproduct of $A, \tau$ the twist map. If

$$
d: M \rightarrow M \otimes M
$$

is the coproduct of $M$, then the following diagrams are commutative

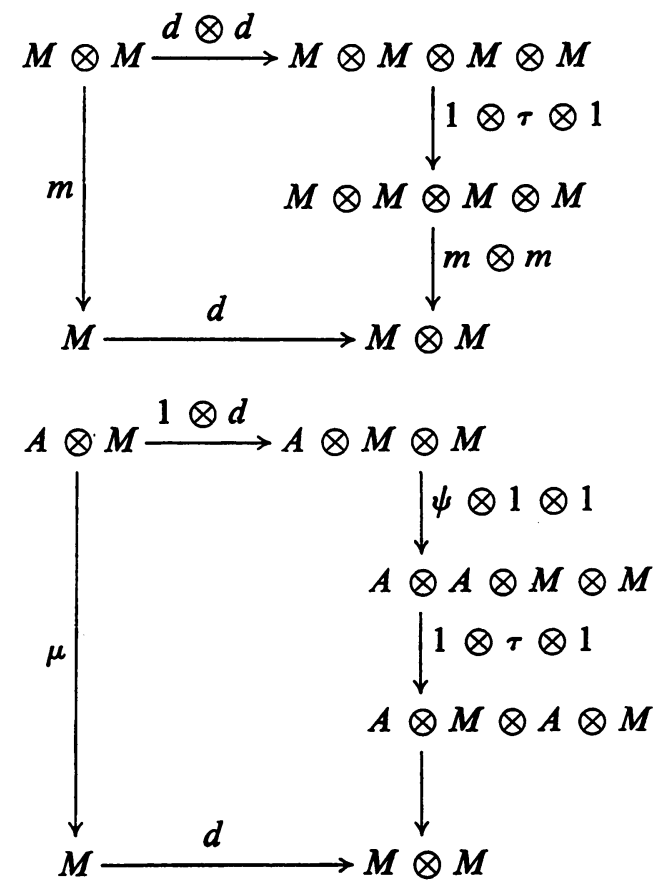

We assume, furthermore, that $M$ and $A$ are finite dimensional over $F$ in each grading.

If we denote by $N_{*}$ the graded dual of $N$, then $A_{*}$ becomes a Hopf algebra with product $\psi_{*}, M_{*}$ becomes a Hopf algebra over $A_{*}$ with coaction $\mu_{*}$, which satisfies:

(C) $\quad\left(1 \otimes m_{*}\right) \circ \mu_{*}=\left(\psi_{*} \otimes 1 \otimes 1\right) \circ(1 \otimes \tau \otimes 1) \circ\left(\mu_{*} \otimes \mu_{*}\right) \circ m_{*}$. 
Suppose $M=F[u]$, a polynomial algebra over $F$ on one generator $u$, $\operatorname{deg} u$ $=s \geqq 1, s$ even if char $F \neq 2$. Then $d(u)=u \otimes 1+1 \otimes u$, and $M_{*}=\Gamma[\bar{u}]$, a divided polynomial algebra [2] on a class $\bar{u}$ of degree $s$. If we denote by $x_{i}$ the class dual to $u^{i}$ in $M_{*_{s}}$, then the multiplication in $M_{*}$ is given by

$$
x_{i} x_{j}=\left(\begin{array}{c}
i+j \\
i
\end{array}\right) x_{i+j}
$$

and the coproduct by

$$
m_{*}\left(x_{n}\right)=\sum_{i+j=n} x_{i} \otimes x_{j}
$$

Let us define the elements $\gamma_{i}^{(n)}$ in $A_{*}$ by

$$
\mu_{*}\left(x_{n}\right)=\sum_{i} \gamma_{i}^{(n)} \otimes x_{i}
$$

We note that $\operatorname{deg} \gamma_{i}^{(n)}=(n-i) s, \gamma_{n}^{(n)}=1, \gamma_{i}^{(n)}=0$ if $i<0$ or $i>n$.

Proposition 1 (VAN DE VeLde). For each $n$ and each pair of integers $s, t$ such that $s+t=i$, we have

$$
\gamma_{i}^{(n)}=\sum_{a} \gamma_{s}^{(a)} \gamma_{t}^{(n-a)}
$$

Proof. Apply formula (C) to $x_{n}$ :

$$
\left(1 \otimes m_{*}\right) \mu_{*}\left(x_{n}\right)=\sum_{i} \gamma_{i}^{(n)}\left(\sum_{j} x_{j} \otimes x_{i-j}\right),
$$

$\left(\psi_{*} \otimes 1 \otimes 1\right)(1 \otimes \tau \otimes 1)\left(\mu_{*} \otimes \mu_{*}\right) m_{*}\left(x_{n}\right)$

$$
\begin{aligned}
& =\left(\psi_{*} \otimes 1 \otimes 1\right)(1 \otimes \tau \otimes 1) \sum_{a}\left(\sum_{s} \gamma_{s}^{(a)} \otimes x_{s}\right)\left(\sum_{t} \gamma_{t}^{(n-a)} \otimes x_{t}\right) \\
& =\sum_{a} \sum_{s, t} \gamma_{s}^{(a)} \gamma_{t}^{(n-a)} \otimes x_{s} \otimes x_{t} .
\end{aligned}
$$

Comparing coefficients:

$$
\gamma_{i}^{(n)}=\sum_{a} \gamma_{s}^{(a)} \gamma_{t}^{(n-a)}
$$

which is the desired formula.

COROLLARY 2. If $\gamma_{0}^{(n)}$ and $\gamma_{1}^{(n)}$ are known for all $n$, then $\gamma_{i}^{(n)}$ can be determined recursively by formula (MF).

2. Projective spaces. Let $M=H^{*}\left(R P^{\infty} ; Z_{2}\right)=Z_{2}[u]$, deg $u=1$; let $A$ be the Steenrod algebra over $Z_{2}$. The elements $\alpha_{i} \in A_{* 2^{4}-1}$ are defined by the equation [4]:

$$
\vartheta u=\left\langle\alpha_{i}, \vartheta\right\rangle u^{2^{i}} \quad \text { for } \vartheta \in A_{2^{i}-1}
$$


It is also known that for all $a \in A_{q}, a u=0$ unless $q=2^{i}-1$, and $a 1=0$ for all $\operatorname{deg} a$ $>0$. Let $x_{i}$ be the dual of $u^{i}$. The equations above imply:

$$
\begin{array}{ll}
\gamma_{0}^{(n)}=0 & \text { for } n>0 \\
\gamma_{1}^{(n)}=0 & \text { if } n \neq 2^{i} \\
\gamma_{1}^{(n)}=\alpha_{i} & \text { if } n=2^{i} .
\end{array}
$$

According to Corollary 2, we can now determine all of the coefficients $\gamma_{i}^{(n)}$ using formula (MF). Several simplifications are possible:

LEMMA 3. For all $n, r$

$$
\begin{aligned}
\gamma_{2^{r}}^{(n)} & =0 \quad \text { if } n \neq 2^{r+i}, \\
& =\alpha_{i}^{2^{r}} \quad \text { if } n=2^{r+i},
\end{aligned}
$$

Proof. The lemma is known for $r=0$. Suppose $r \geqq 1$, let $s=2^{r-1}, t=2^{r-1}$ in formula (MF) and notice that all terms cancel out in pairs, except the term $\gamma_{2^{r}-1}^{(k)} \gamma_{2^{r}-1}^{(k)}$ if $n=2 k$.

COROllaRy 4 (Periodicity). For all $r \geqq 0, i \geqq 0$, and $n<2^{r+1}-2^{r}$,

$$
\gamma_{i+2^{r}}^{(n+2)}=\gamma_{i}^{(n)} \text {. }
$$

Proof. According to formula (MF)

$$
\gamma_{i+2^{r}}^{(n+2)}=\sum_{a} \gamma_{i}^{\left(n+2^{r}-a\right)} \gamma_{2^{r}}^{(a)}
$$

However, $\gamma_{2^{r}}^{(0)}=0, \gamma_{2^{r}}^{(2)}=1$, and $\gamma_{2^{r}}^{(a)}=0$ for $2^{r}<a<2^{r+1}$; since $n<2^{r+1}-2^{r}, n+2^{r}$ $<2^{r+1}$, thus $a<2^{r+1}$, and the formula reduces to

$$
\gamma_{i+2^{r}}^{(n+2)}=\gamma_{i}^{(n)} \text {. }
$$

Corollary 4 asserts that if we plot $\gamma_{i}^{(n)}$ in a table with respect to $n, i$, then the resulting table is triangular, has entries 1 along the line $n=i$, and is periodic near the diagonal with period $2^{r}$ (the periodicity being a $2^{r} \times 2^{r}$ matrix). The periodic blocks

\begin{tabular}{|c|c|}
\hline & 12 \\
\hline 3 & \\
\hline & \\
\hline
\end{tabular}
$B_{r}$ for $r \leqq 4$ are given in the first sequence of tables. To simplify the tables, we notice that Corollary 4 implies that

$$
B_{r+1}=\left(\begin{array}{ll}
B_{r} & 0 \\
C_{r} & B_{r}
\end{array}\right)
$$

hence we will only give the matrices $C_{r}$.

TABle I. $C_{1}$

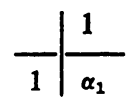

TABLE II. $C_{2}$ 
TABLE III. $C_{3}$

\begin{tabular}{c|cccc} 
& 1 & 2 & 3 & 4 \\
\hline 5 & 0 & 0 & $\alpha_{1}^{2}$ & 0 \\
6 & 0 & 0 & $\alpha_{1}^{3}+\alpha_{2}$ & 0 \\
7 & 0 & 0 & 0 & 0 \\
8 & $\alpha_{3}$ & $\alpha_{2}^{2}$ & $\alpha_{2} \alpha_{1}^{2}$ & $\alpha_{1}^{4}$
\end{tabular}

TABLE IV. $C_{\boldsymbol{4}}$

\begin{tabular}{r|cccccccc} 
& 1 & 2 & 3 & 4 & 5 & 6 & 7 & 8 \\
\hline 9 & 0 & 0 & $\alpha_{2}^{2}$ & 0 & $\alpha_{1}^{4}$ & 0 & $\alpha_{1}^{2}$ & 0 \\
10 & 0 & 0 & $\alpha_{2}^{2} \alpha_{1}+\alpha_{3}$ & 0 & $\alpha_{1}^{5}$ & $\alpha_{1}^{4}$ & $\alpha_{1}^{3}+\alpha_{2}$ & 0 \\
11 & 0 & 0 & 0 & 0 & 0 & 0 & $\alpha_{1}^{4}$ & 0 \\
12 & 0 & 0 & $\alpha_{2}^{3}+\alpha_{3} \alpha_{1}^{2}$ & 0 & $\alpha_{2} \alpha_{1}^{4}+\alpha_{3}$ & $\alpha_{1}^{6}+\alpha_{2}^{2}$ & $\alpha_{1}^{5}+\alpha_{2} \alpha_{1}^{2}$ & 0 \\
13 & 0 & 0 & 0 & 0 & 0 & 0 & $\alpha_{1}^{6}+\alpha_{2}^{2}$ & 0 \\
14 & 0 & 0 & 0 & 0 & 0 & 0 & $\alpha_{1}^{7}+\alpha_{2} \alpha_{1}^{4}+\alpha_{2}^{2} \alpha_{1}+\alpha_{3}$ & 0 \\
15 & 0 & 0 & 0 & 0 & 0 & 0 & 0 & 0 \\
16 & $\alpha_{4}$ & $\alpha_{3}^{2}$ & $\alpha_{3} \alpha_{2}^{2}$ & $\alpha_{2}^{4}$ & $\alpha_{3} \alpha_{1}^{4}$ & $\alpha_{2}^{2} \alpha_{1}^{4}$ & $\alpha_{2} \alpha_{1}^{6}+\alpha_{2}^{3}+\alpha_{3} \alpha_{1}^{2}$ & $\alpha_{1}^{8}$
\end{tabular}

3. The coaction on $x_{128}$. We recall that $H_{*}\left(R P^{\infty}\right)$ is, as an algebra, an exterior algebra on the elements $x_{2}$, for $r=0,1,2, \ldots$. Since $\mu_{*}$ is a homomorphism of algebras, to determine $\mu_{*}\left(x_{n}\right)$ it is sufficient to know $\mu_{*}\left(x_{2^{r}}\right)$ for $r$ entering in the dyadic expansion of $n$. The computation of $\mu_{*}\left(x_{n}\right)$ for $n \leqq 128$ was carried out on the IBM 7094. The results for $n=32$ are listed below. In the table below each line is headed by $j$; after the colon $\gamma_{j}^{(32)}$ follows.

TABLE V. Coaction on $x_{32}$

0: 0

16: $\alpha_{1}^{16}$.

1: $\alpha_{5}$

17: $\alpha_{4}$.

2: $\alpha^{2}$.

18: $\alpha_{3}^{2}$.

3: $\alpha_{4} \alpha_{3}^{2}$.

19: $\alpha_{3} \alpha_{2}^{2}$.

4: $\alpha_{3}^{4}$.

20: $\alpha_{2}^{4}$.

5: $\alpha_{4} \alpha_{2}^{4}$.

21: $\alpha_{3} \alpha_{1}^{4}$.

6: $\alpha_{3}^{2} \alpha_{2}^{4}$.

22: $\alpha_{2}^{2} \alpha_{1}^{4}$.

7: $\alpha_{4} \alpha_{2}^{2} \alpha_{1}^{4}+\alpha_{3}^{3} \alpha_{1}^{4}+\alpha_{3} \alpha_{2}^{6}$.

23: $\alpha_{3} \alpha_{1}^{2}+\alpha_{2}^{3}+\alpha_{2} \alpha_{1}^{6}$.

8: $\alpha_{2}^{8}$.

24: $\alpha_{1}^{8}$.

9: $\alpha_{4} \alpha_{1}^{8}$.

25: $\alpha_{3}$.

10: $\alpha_{3}^{2} \alpha_{1}^{8}$.

26: $\alpha_{2}^{2}$.

11: $\alpha_{4} \alpha_{2}^{2}+\alpha_{3}^{3}+\alpha_{3} \alpha_{2}^{2} \alpha_{1}^{8}$.

27: $\alpha_{2} \alpha_{1}^{2}$.

12: $\alpha_{2}^{4} \alpha_{1}^{8}$.

28: $\alpha_{1}^{4}$.

13: $\alpha_{4} \alpha_{1}^{4}+\alpha_{3} \alpha_{2}^{4}+\alpha_{3} \alpha_{1}^{12}$.

29: $\alpha_{2}$.

14: $\alpha_{3}^{2} \alpha_{1}^{4}+\alpha_{2}^{6}+\alpha_{2}^{2} \alpha_{1}^{12}$.

30: $\alpha_{1}^{2}$.

15: $\alpha_{4} \alpha_{1}^{2}+\alpha_{3}^{2} \alpha_{2}+\alpha_{3} \alpha_{2}^{2} \alpha_{1}^{4}+\alpha_{2}^{5} \alpha_{1}^{2}$

31. $\alpha_{1}$. $+\alpha_{3} \alpha_{1}^{10}+\alpha_{2}^{3} \alpha_{1}^{8}+\alpha_{2} \alpha_{1}^{14}$.

32: 1 .

4. Classifying spaces and Thom spaces. Let $O(n)$ be the orthogonal group in dimension $n$ and $O=\lim _{n \rightarrow \infty} O(n)$. Then $B O(1)=R P^{\infty}$, and $O(1) \rightarrow O$ induces 
$B O(1) \stackrel{b}{\rightarrow} B O$, which is a monomorphism in homology with $Z_{2}$ coefficients. Furthermore, $H_{*}\left(B O ; Z_{2}\right)$ is a polynomial algebra [6], [3] on classes $u_{i} \in H_{i}\left(B O ; Z_{2}\right)$ defined by

$$
\left\langle u_{i}, w_{1}^{i}\right\rangle=1, \quad\left\langle u_{i}, m\right\rangle=0,
$$

where $m$ is any monomial in $w_{j}$ not equal to $w_{1}^{i}$. Thus $j_{*}\left(x_{i}\right)=u_{i}$, hence we know the coaction on $u_{i}$.

The homology of $B O(n)$ is a subcoalgebra of the homology of $B O$ :

PROPOsition 5. $H_{*}(B O(n))$ is the vector subspace of $Z_{2}\left[x_{1}, x_{2}, \ldots\right]$ with basis all monomials having $\leqq n$ products of generators.

Proof. Immediate, using a counting argument.

EXAMPLE. $H_{*}(B O(2))$ is given in low dimensions by linear combinations of 01.

$1 x_{1}$.

$2 x_{1}^{2}, x_{2}$.

$3 x_{2} x_{1}, x_{3}$.

$4 x_{2}^{2}, x_{3} x_{1}, x_{4}$.

Let the Thom space $M O(n)$ be the mapping cone of $B O(n-1) \rightarrow B O(n)$.

PROPOSITION 6. $\tilde{H}_{*}(M O(n))$ is the vector subspace of $Z_{2}\left[x_{1}, \ldots, x_{n}, \ldots\right]$ having as a basis all monomials consisting of precisely $n$ factors.

Proof. $\tilde{H}_{*}(M O(n))$ is the quotient of $H_{*}(B O(n))$ by $H_{*}(B O(n-1))$. We notice that $\tilde{H}_{*}(M O(n))$ is actually a direct summand of $H_{*}(B O(n))$ as an $A_{*}$-comodule, since $\mu_{*}$ preserves the number of products of generators occurring in a monomial. This remark together with the proposition proves:

Proposition 7.

$$
\tilde{H}_{*}(B O) \cong \underbrace{\infty}_{n=1} \tilde{H}_{*}(M O(n))
$$

as $A_{*}$-comodules.

Proposition 8. The map in homology induced by

$$
M O(m) \wedge M O(n) \rightarrow M O(m+n)
$$

is the restriction of the product in $H_{*}(B O)$.

Let $M O$ be the spectrum $\{M O(n)\}$, where the maps $S^{1} \wedge M O(n) \rightarrow M O(n+1)$ are given by

$$
S^{1} \wedge M O(n) \rightarrow M O(1) \wedge M O(n) \rightarrow M O(n+1) .
$$


In homology $H_{*}\left(M O(n) ; Z_{2}\right) \stackrel{g_{n}}{\rightarrow} H_{*}\left(M O(n+1) ; Z_{2}\right)$ is given by

$$
g_{n}(a)=x_{1} \cdot a
$$

The homology of $M O$ with $Z_{2}$ coefficients is defined to be

$$
H_{i}(M O)=\operatorname{inj} \lim H_{n+i}(M O(n)) .
$$

Thus $H_{i}(M O) \cong H_{i}(B O)$ as vector spaces, though not as $A_{*}$-comodules. We also note that the maps

$$
M O(m) \wedge M O(n) \rightarrow M O(m+n)
$$

make $H_{*}\left(M O ; Z_{2}\right)$ into an algebra over $A_{*}$.

Proposition 9.

$$
H_{*}\left(M O ; Z_{2}\right) \cong Z_{2}\left[a_{1}, a_{2}, \ldots\right]
$$

and the coaction of $A_{*}$ on $a_{n}$ is obtained by considering $\mu_{*}\left(x_{n+1}\right)$ and replacing $x_{m}$ by $a_{m-1}$. Furthermore, $a_{n}$ is represented by the element $x_{1}^{m} x_{n+1}$, where $m$ is large.

Proof. Immediate.

We list the coactions of the first few generators:

$$
\begin{aligned}
\mu_{*}(1)= & 1 \otimes 1, \\
\mu_{*}\left(a_{1}\right)= & \alpha_{1} \otimes 1+1 \otimes a_{1}, \\
\mu_{*}\left(a_{2}\right)= & 1 \otimes a_{2}, \\
\mu_{*}\left(a_{3}\right)= & \alpha_{2} \otimes 1+\alpha_{1}^{2} \otimes a_{2}+\alpha_{1} \otimes a_{1}+1 \otimes a_{3}, \\
\mu_{*}\left(a_{4}\right)= & \alpha_{1}^{2} \otimes a_{2}+1 \otimes a_{4}, \\
\mu_{*}\left(a_{5}\right)= & \left(\alpha_{1}^{3}+\alpha_{2}\right) \otimes a_{2}+\alpha_{1} \otimes a_{4}+1 \otimes a_{5}, \\
\mu_{*}\left(a_{6}\right)= & 1 \otimes a_{6} \\
\mu_{*}\left(a_{7}\right)= & \alpha_{3} \otimes 1+\alpha_{2}^{2} \otimes a_{1}+\alpha_{2} \alpha_{1}^{2} \otimes a_{2}+\alpha_{1}^{4} \otimes a_{3}+\alpha_{2} \otimes a_{4}+\alpha_{1}^{2} \otimes a_{5} \\
& +\alpha_{1} \otimes a_{6}+1 \otimes a_{7} .
\end{aligned}
$$

We note that Proposition 9 corrects formula (3.27) in [3] (this was brought to our attention in a letter by J. C. Su; the proof of the main theorem in [3] goes through without change).

The results for $H_{*}(B O)$ and $H_{*}(M O)$ immediately go over to results in $H_{*}(B U)$, $H_{*}(M U), H_{*}(B S p), H_{*}(M S p)$, for the maps

$$
\begin{gathered}
H_{*}(B O) \stackrel{f}{\longrightarrow} H_{*}(B U) \stackrel{g}{\longrightarrow} H_{*}(B S p), \\
H_{*}(M O) \stackrel{h}{\longrightarrow} H_{*}(M U) \stackrel{k}{\longrightarrow} H_{*}(M S p),
\end{gathered}
$$

and the kernels are well known: 
Proposition 10.

(1) $\operatorname{Ker} f=$ ideal generated by $x_{2 i+1}$, all $i$;

(2) $H_{*}(B U)=Z_{2}\left[y_{1}, y_{2}, \ldots\right]$, deg $y_{i}=2 i$, and $y_{i}=f\left(x_{2 i}\right)$;

(3) Ker $g=$ ideal generated by $y_{2 i-1}$, all $i$;

$$
H_{*}(B S p)=Z_{2}\left[z_{1}, z_{2}, \ldots\right] \text {, deg } z_{i}=4 i \text {, and } z_{i}=g\left(y_{2 i}\right) \text {. }
$$

(4) If in (1), (2), (3) we replace $B$ by $M, f$ by $h, g$ by $k, x_{i}$ by $a_{i}, y_{i}$ by $b_{i}, z_{i}$ by $c_{i}$, then the statements remain true.

\section{REFERENCES}

1. J. F. Adams, On formulas of Thom and Wu, Proc. London Math. Soc. (3) 11 (1961), 741-752.

2. N. E. Steenrod, Cohomology operations (lectures by N. E. Steenrod written and revised by D. B. A. Epstein), Annals of Mathematics Studies No. 50, Princeton Univ. Press, Princeton, N. J., 1962.

3. A. Liulevicius, A proof of Thom's theorem, Comment. Math. Helv. 37 (1962), 121-131.

4. J. Milnor, The Steenrod algebra and its dual, Ann. of Math. 67 (1958), 150-171.

5. R. Van de Velde, An algebra of symmetric functions and applications, Thesis, Univ. of Chicago, Chicago, Ill., 1967.

6. C. T. C. Wall, Determination of the cobordism ring, Ann. of Math. 72 (1960), 292-311.

THE UNIVERSITY OF Chicago,

Chicago, Illinois 